\title{
Editorial
}

\section{Soul, Spirit, and Consciousness in Psychology}

\section{and Philosophy}

\section{Traditions, Current Views, Perspectives}

\author{
Ulrich W. Weger ${ }^{1,2}$ and Johannes Wagemann ${ }^{3}$ \\ ${ }^{1}$ Department for Psychology and Psychotherapy, University of Witten/Herdecke, Germany \\ 2 School of Psychology, University of Kent at Canterbury, UK \\ ${ }^{3}$ Institute for Waldorf Education, Inclusion and Interculturalism, Alanus University, Campus Mannheim, Germany
}

The question about body, soul, and spirit has been part of most contemplative traditions over the millennia and has likewise been at the center of philosophical and theological enquiries both inside academia and beyond. Since its inception as an academic discipline, psychology has emancipated itself more and more from this tradition: while the concepts still resonated amongst some of the early pioneers of the field, they receded more and more into the background of scholarly debate. Other questions have emerged into prominence - some of them perhaps bearing a certain resemblance to - or at least unfolding in a succession of - the original trichotomy; and yet, many core questions remain a challenge or even appear intractable on the basis of the current research framework - including the question about phenomenological consciousness, the self, the mind-body problem, and others (see also Beauregard et al., 2018; Sparby, 2021; Weger \& Wagemann, 2015).

In addressing these issues, new and creative approaches are needed. The methods and techniques currently available in the cognitive and behavioral sciences seem to be ideally suited to explore phenomena in the outer/behavioral world. But the conundrums just mentioned also involve an inner, "genuinely" psychological dimension that is difficult to make sense of when conceptualized merely as an appendix to the outer/material realm. Outer microscopes are thus not sufficient. Unless we are tempted to abandon the conceptual issues/questions to begin with, different methodological approaches are needed - and perhaps these include the use of inner microscopes which allow for qualitatively new conceptual insights (see also Wagemann et al., 2018). Different contemplative, therapeutical, and folk-psychological traditions have pursued such an inward observational approach - and even in academic enquiry such a methodology is gaining more and more recognition - though mostly as a niche topic.

Is it meaningful to illuminate the question about body, soul, and spirit in a context where it is typically seen as an anachronism - and often enough even seen as a nonacademic way of thinking? We are convinced it is - and we consider it not only meaningful but also urgent; at the very least we should not a priori disregard possible alternatives as long as those earlier questions appear intractable from the contemporary perspective (cf. Majorek, 2012).

In the current issue, we sought to bring this theme back to the center of psychological enquiry and proposed a special section in the European Psychologist. It appeared a suitable outlet due to its multidisciplinary approach, its openness to non-traditional research trends - and because the theme also has a certain history within the European thought tradition, for instance during the era of classic Greek (Aristotelian) philosophy or German Idealism (Schelling, Fichte, Hegel, Novalis, and others).

Of the submitted manuscripts - stemming from a range of different disciplines - two passed the review process and are included in this special section. In the first, Gutland (2021) points to the need to anchor conceptual validity directly in human experience; he relates his enquiry into conscious awareness to experiences from the sense world as well as from the conceptual realm (thought, reasoning, etc.) and hints on the quality of essences as non-sensory mental (conceptual) events; he sets out to distinguish experiences from the sense world and the conceptual 
world phenomenologically and identifies parallels that prompt insights into the understanding of consciousness at large.

In the second article, Weger et al. (2021) explore facets of a dichotomic (body-soul) versus a trichotomic (body-soulspirit) conceptualization of human nature. The authors distinguish between the tendency to universalize vs. the tendency to individualize and thereby arrive at an understanding of body, soul, and spirit that puts body (individualization) and spirit (universalization) at the two poles of the continuum. In between they place the soul as a balancing entity - and as an entrance to a phenomenology that mediates between both sides. A bridge between both articles is the sensory-individualizing link on the one hand - and the conceptual-universalizing link on the other.

In our initial theme call, four core questions were raised: How can we characterize and distinguish the concepts soul, spirit, and consciousness? What are appropriate methods to investigate consciousness and can (aspects of) soul and spirit be researched scientifically? Both issues were at least in part spoken to in the two articles. The other two, for now, await further enquiry: How are these domains understood from a lay perspective, or in pre-scientific forms of thinking? And how does an understanding of soul, spirit, or consciousness vary across cultures and how has it evolved throughout the history of science?

We acknowledge that we had expected a more substantial number of contributions - but the outcome is certainly also an indication that the theme is by no means firmly established within the academic landscape. For example, research on spirituality is constantly increasing - with more than 5,000 outputs over the past decade alone (in response to entering the search term "spirituality" on the "Web of Knowledge" database on November 19, 2020); by now there is even an impact-factor ranked journal with the word in the title (Journal of Religion and Spirituality) and related concepts such as mindfulness or meditation have a veritable history in contemplative traditions. And yet, where addressed in contemporary psychological enquiry, these topics are typically treated as objects of enquiry; by contrast, there is little research on how we can fine-tune and calibrate our consciousness, for instance through the instrument of mindfulness or other first-person methods, although one might argue that this would be a useful complementary approach to uncover psychological phenomena that are not accessible to conventional analysis. This line of enquiry still bears the potential for future research. Equally important, a connection to other research disciplines (philosophy, medicine, ethics, and quantum physics) would be beneficial in order to connect to similar questions and research traditions in these related realms.

\section{References}

Beauregard, M., Trent, N. L., \& Schwartz, G. E. (2018). Toward a postmaterialist psychology: Theory, research, and applications. New Ideas in Psychology, 50, 21-33. https://doi.org/10.1016/ j.newideapsych.2018.02.004

Gutland, C. (2021). Psychological consciousness of non-psychological contents: Aspects of a phenomenology of sensations and thoughts. European Psychologist, 26(2), 73-84. https://doi. org/10.1027/1016-9040/a000426

Sparby, T. (2021). Meditation and spiritual practice. Religions, 12(Special Issue).

Majorek, M. (2012). Does the brain cause conscious experience? Journal of Consciousness Studies, 19(3-4), 121-144.

Wagemann, J., Edelhäuser, F., \& Weger, U. (2018). Outer and inner dimensions of brain and consciousness - refining and integrating the phenomenal layers. Advances in Cognitive Psychology, 14, 167-185. https://doi.org/10.5709/acp-0248-2

Weger, U., Sparby, T., \& Edelhäuser, F. (2021). Dualistic and trichotomic approaches in psychological enquiry. The question about body, soul, and spirit. European Psychologist, 26(2), 85-95. https://doi.org/10.1027/1016-9040/a000427

Weger, U. W., \& Wagemann, J. (2015). The behavioral, experiential and conceptual dimensions of psychological phenomena: Body, soul and spirit. New Ideas in Psychology, 39, 23-33. https://doi. org/10.1016/j.newideapsych.2015.07.002

Published online May 7, 2021

\section{Ulrich Weger}

Department for Psychology and Psychotherapy \&

Institute for First-Person Research

University of Witten/Herdecke

Alfred-Herrhausen-Str. 50

58448 Witten

Germany

ulrich.weger@uni-wh.de

\author{
Johannes Wagemann \\ Alanus University \\ Campus Mannheim \\ Am Exerzierplatz 21 \\ 68169 Mannheim \\ Germany \\ johannes.wagemann@alanus.edu
}

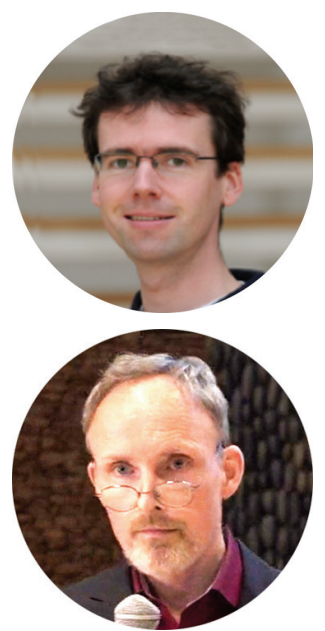

Ulrich Weger (PhD) is professor of foundations in psychology at the Department of Psychology and Psychotherapy, University of Witten/ Herdecke, who specializes in firstperson research.

Johannes Wagemann (PhD) is professor for consciousness research at the Institute of Waldorf Education, Inclusion, and Interculturality at the Alaunus University, Mannheim. 